PACS 02.70.-c, 02.70.Ns, 61.20.Ja, 82.65.Yh

\title{
IONIC HYDRATION NEAR A PLATINUM(100) SURFACE
}

\author{
K.HEINZINGER \\ Max-Planck-lnstitut fur Chemie (Otto-Hahn-lnstitut), \\ D-55020 Mainz, Germany
}

Received September 4, 1997

\begin{abstract}
In the Molecular Dynamic simulations reported here the flexible BJH model is used for the description of water-water interactions. The potentials for the interactions between ions and water, platinum surface and water as well as platinum surface and ions are based on $\mathrm{ab}$ initio calculations. It is demonstrated that the structural and dynamical properties of the ions and their hydration shells in the layer adsorbed on the $\mathrm{Pt}(100)$ surface are significantly different from those in a bulk solution. But the influence of the metal on these properties is practically limited to the adsorbed layer.
\end{abstract}

\section{Introduction}

It seems to be straightforward to write down the potentials which describe the interactions between electrolyte solutions and metal surfaces. At least three contributions are necessary. Two of them - the repulsive and the dispersion term - can simply be described by a Lennard-Jones potential. For the third term - the Coulomb interactions - the image charge model is available. It states that the Coulomb interaction between a charge and a metal surface can be described in such a way that an additional charge with the same amount but of the opposite sign is imagined the position of which is determined as the mirror image of the original charge where the surface acts as a mirror. This rule is not only valid for ions but also for the partial charges which describe the dipole moment of the water molecule.

The simulation of water near a $\operatorname{Pt}(100)$ surface with such potentials leads to disagreement with the experiment. The potential drop at a water metal interface is a property which is easily measurable. Its value is $1.1 \mathrm{~V}$ for the $\operatorname{Pt}(100)$ surface. This potential drop can also easily be calculated from the simulation through the orientation of water molecules:

$$
\chi=\varepsilon_{0} \int \mathrm{d} z \rho_{\mu}(z) .
$$

Here $\rho_{\mu}(z)$ denotes the dipole density as a function of the distance $z$ from the surface. As the simulation leads to an orientation of the dipole moments preferentially parallel to the surface and as the distribution of the orientations is found to be symmetric this means that the potential drop is equal to zero, in contradiction to the experiment.

This discrepancy must result from the image charge model. It has two weak points. Firstly, the metal surface is assumed to be uniform. This can only be correct if a water molecule or an ion is sufficiently far away from. the surface. As soon as the distance is reduced to only a few $\AA$ then the

(C) K.Heinzinger, 1997

ISSN 0452-9910. Condensed Matter Physics 1997 No 11 (3-15) 
water molecule or the ion will see the metal atoms itself and a corrugation of the potentials is necessary for the correct description. Secondly, the image charge model is not specific for a metal. The specificity of the metal can only be introduced by $\varepsilon$ and $\sigma$ in the LJ potential. This does not seem to be sufficient. In order to prevent these difficulties it appears to be reasonable not to use the image charge model and the LJ potential but derive the total potential from quantum mechanical calculations.

On the basis of ab initio calculations between a platinum cluster and a water molecule [1] a potential was developed which has led to good agreement with the measured potential drop [2]. The results of simulations with such a potential in respect to the structure and the dynamics of pure water between two $\mathrm{Pt}(100)$ surfaces have been reported in detail in [3,4]. Additional simulations with applied external electrical fields were performed [5]. The results of these simulations of pure water near a $\mathrm{Pt}(100)$ surface have been reviewed recently [6]. In this paper results on the influence of the (100) surface on the properties of a lithium and an iodide ion are reported.

\section{Potentials and simulations}

The atoms at the $\operatorname{Pt}(100)$ surface are arranged quadratically with a lattice constant of $2.77 \AA$ (figure 1). For the $a b$ initio calculations the metal surface is approximated by a cluster which consists of five atoms for the

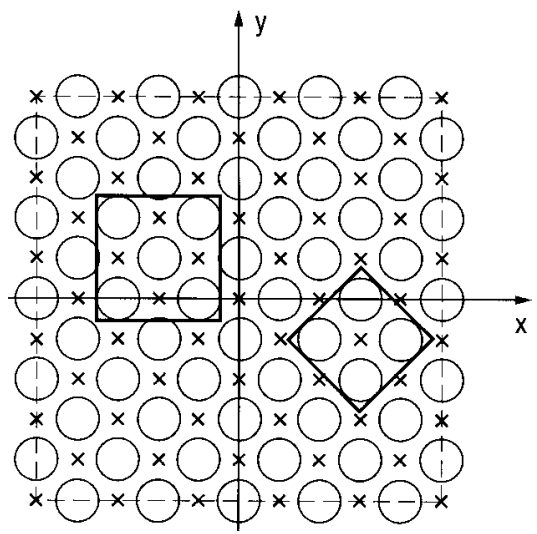

Figure 1. Sketch of the arrangement of platinum atoms in the first (circles) and second (crosses) layer of the $\mathrm{Pt}(100)$ surface which coincides with the $x y$ plane of the basic periodic cube the border of which is marked by dashed lines. Form and size of the two clusters used for the ab initio calculations are indicated. position) or between four platinum atoms (h-position) of the surface. Figure $2 \mathrm{~b}$ shows that the adsorption energy is significantly smaller if - by keeping the t-position - the dipole moment vector is directed towards the surface. The potential energy for an orientation where the dipole and of nine for the platinum- $\mathrm{I}^{-}$interactions $[1,7]$. The platinum atoms in the clusters are arranged according to the $\mathrm{Pt}(100)$ surface. In the five-atomic cluster four atoms belong to the first and one atom to the second layer while in the case of the nine-atomic cluster the corresponding numbers are five and four (figure 1). The results of these calculations are presented in figures 2-4.

The

potential energy of water molecules depends - besides on the distance from the surface - also upon their position relative to the platinum atoms of the first layer and upon their orientations. It can be seen from figure $2 \mathrm{a}$ that the potential energy is the lowest for a position of the oxygen atom on top of a platinum, atom of the first layer (t-position) and an orientation where the dipole moment vector points away from the surface. For the same orientation only a small difference is found for relative positions where the oxygen atom is positioned between two platinum, atoms (b- 
moment vector is parallel to the surface is found between the other two orientations in the whole distance range. For this orientation the energy depends also on the angle between the plane of the water molecule and the surface. For distances larger than about $6 \AA$ from the surface the potential energy does no longer depend on the relative position and the orientation of water molecules. For such distances the image charge model might be valid.

The corrugation of the surface in respect to the potential energy is depicted in figure 3 . For the calculation of this figure for each point in the $x y$-plane of the basic cube the distance $z$ from the surface is chosen such that the energy has a minimum. The dipole moment vector points away from the surface. The numbers indicate the coordinates of the platinum atoms of the first layer. By the comparison with figure 1 it becomes clear that the potential minima coincide with the positions of the platinum atoms of the first layer in accordance with figure 2 .

The potential energy of $\mathrm{Li}^{+}$and an $\mathrm{I}^{-}$as a function of distance from the surface for the three positions t, b, and $\mathrm{h}$ is depicted in figure 4. Different from the water case the potential minima for the ions are

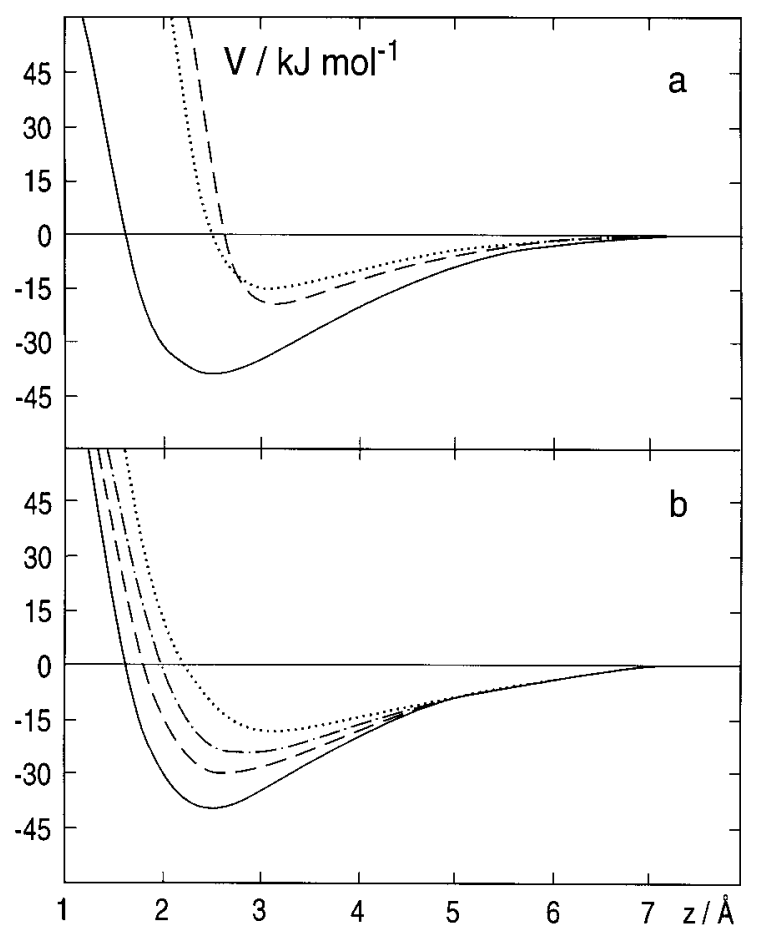

Figure 2. Potential energy of a water molecule as a function of distance of the oxygen atom from an infinitely extended Pt(100) surface, (a) For water on top of a metal atom (full line), on a bridge site (dashed), and on a fourfold hollow site (dotted). The dipole moment vector of water molecule points away from the surface in all three cases, (b) For different orientations of the dipole moment vector relative to the surface: pointing away (full), towards (dotted), and parallel with the proton-proton vector parallel (dashed) and perpendicular (dash-dotted) to the surface. In all four cases the adsorption site is on top of a metal atom [3).

found for h-positions while the t-positions are energetically least favourable. The adsorption energy of $\mathrm{I}^{-}$is almost by a factor of ten larger than that of a water molecule. The corrugation of the potentials is not presented in a figure. It is very similar to that shown in figure 3, only maxima and minima are more pronounced and exchanged.

The tetragonal basic box for the MD simulations of pure water and a 2.2 molal Lil solution at the $\operatorname{Pt}(100)$ surface is sketched in figure 5 . The space between the five layers of platinum atoms at each side is sufficient for about six layers of water molecules. In agreement with the lattice constant of the 
(100) surface the following side lengths of the box result: $L_{x}=L_{y}=19.6 \AA$ and $L_{z}=45 \AA$. The number of 305 water molecules between the platinum

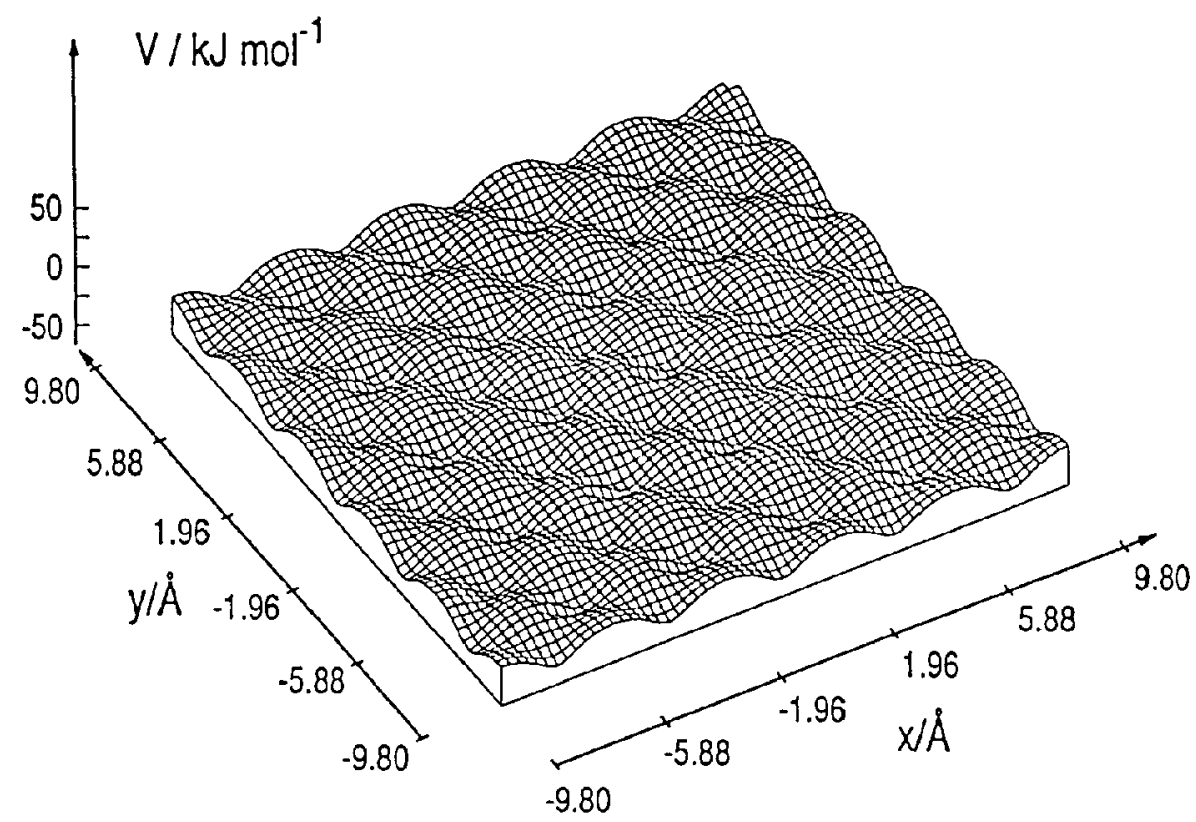

Figure 3. Adsorption energy of a water molecule at an infinitely extended $\operatorname{Pt}(100)$ surface as a function of $x$ and $y$. The distance $z$ from the surface is chosen in such a way that the energy has a minimum. The dipole moment vector points away from the surface [3].

surfaces is found in such a way that at the beginning of the simulation water molecules were added until the density of pure water was reached in the center of the box. In this way the usual experimental conditions are realized. In the case of the electrolyte solution with 298 water molecules, $10 \mathrm{Li}^{+}$and $10 \mathrm{I}^{-}$a 2.2 molal solution resulted. In the $x$ - and $y$-directions periodic boundary conditions were introduced, so that an infinitely extended water lamella between platinum surfaces was simulated. As long as no external electrical field is applied both surfaces are equivalent and the comparison of the calculated properties is a measure of statistical significance $[3,8]$.

The water-water interaction is described by the BJH model [9] and the ion-water potentials are derived from $a b$ inition calculations [10]. For the long range Coulomb interactions the Ewald method is used, modified for two-dimensional systems. For all other interactions the "shifted force method" is employed [11].

\section{Results and discussion}

\subsection{Density profiles and the orientation of the water molecules}

The first property to be calculated when the influence of the metal surface on the structure of water is investigated is the density of the oxygen and hydrogen atoms as a function of distance from the surface. Both density profiles are depicted in figure 6 . In the first layer the density of $\mathrm{O}$ is almost four times and that of $\mathrm{H}$ three times as high as in bulk water, 
a consequence of the strong interaction of water molecules with platinum atoms (figure 2). Second, but already much less pronounced, density maxima are found at $5.5 \AA$. For distances larger than $9 \AA$ the density is no

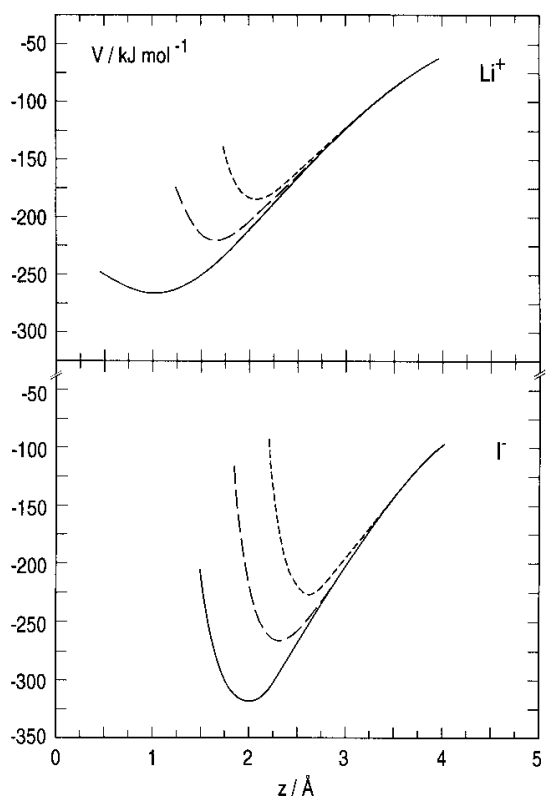

Figure 4. Potential energy of a lithium and an iodide ion as function of distance from an infinitely extended $\mathrm{Pt}(100)$ surface for ion positions on top of a platinum atom (- - ), on a bridge site ($-)$ and a hollow site - $[7]$.

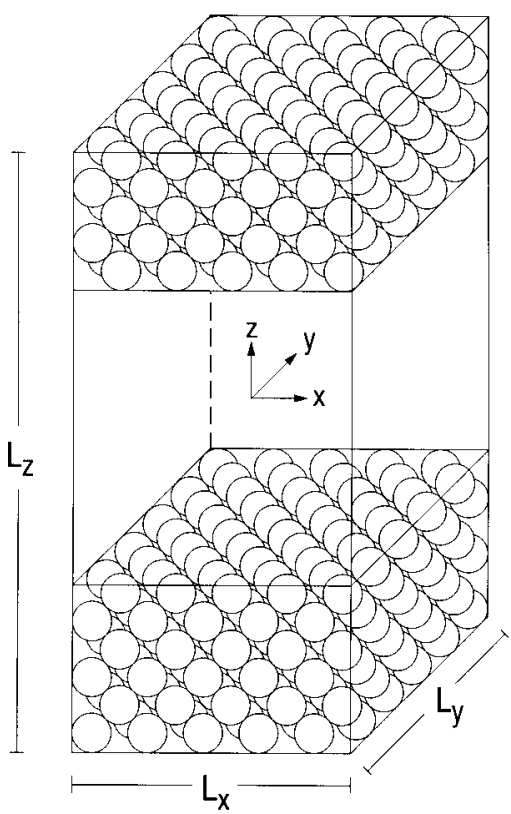

Figure 5. Sketch of the basic tetragonal simulation cell. The water molecules and the ions are located in the center of the box, and the platinum atoms are represented by the circles. The coordinate system which is used troughout the paper is defined in the center. longer influenced by the surface. The same distance for the first maxima in the $\mathrm{O}$ and $\mathrm{H}$ density profiles demonstrates that the dipole moment vector in the adsorbed layer is preferentially orientated parallel to the surface. The surplus of positive or negative charges in the distance range smaller than $7 \AA$ indicates a potential drop different from zero.

For the calculation of the dipole moment density as a function of distance from the surface and, from it, the potential drop according to equation (1), the orientation of water molecules has been calcu-

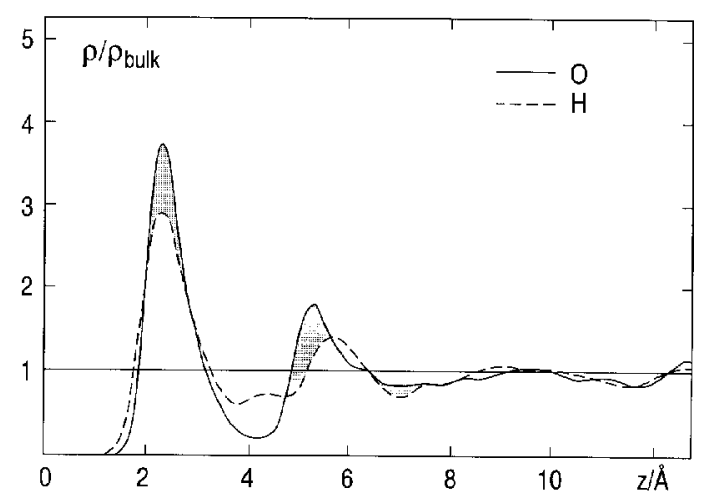

Figure 6. Normalized oxygen and hydrogen atom densities as a function of distance from the $\operatorname{Pt}(100)$ surface. The shaded areas indicate an excess of negative charges [6]. 
lated separately for distance ranges of about $1 \AA$. The distributions of $\cos \vartheta_{\mu}$, where $\vartheta_{\mu}$ denotes the angle between the dipole moment vector of the water molecule and the vector normal to the surface, are shown in figure 7 (left).
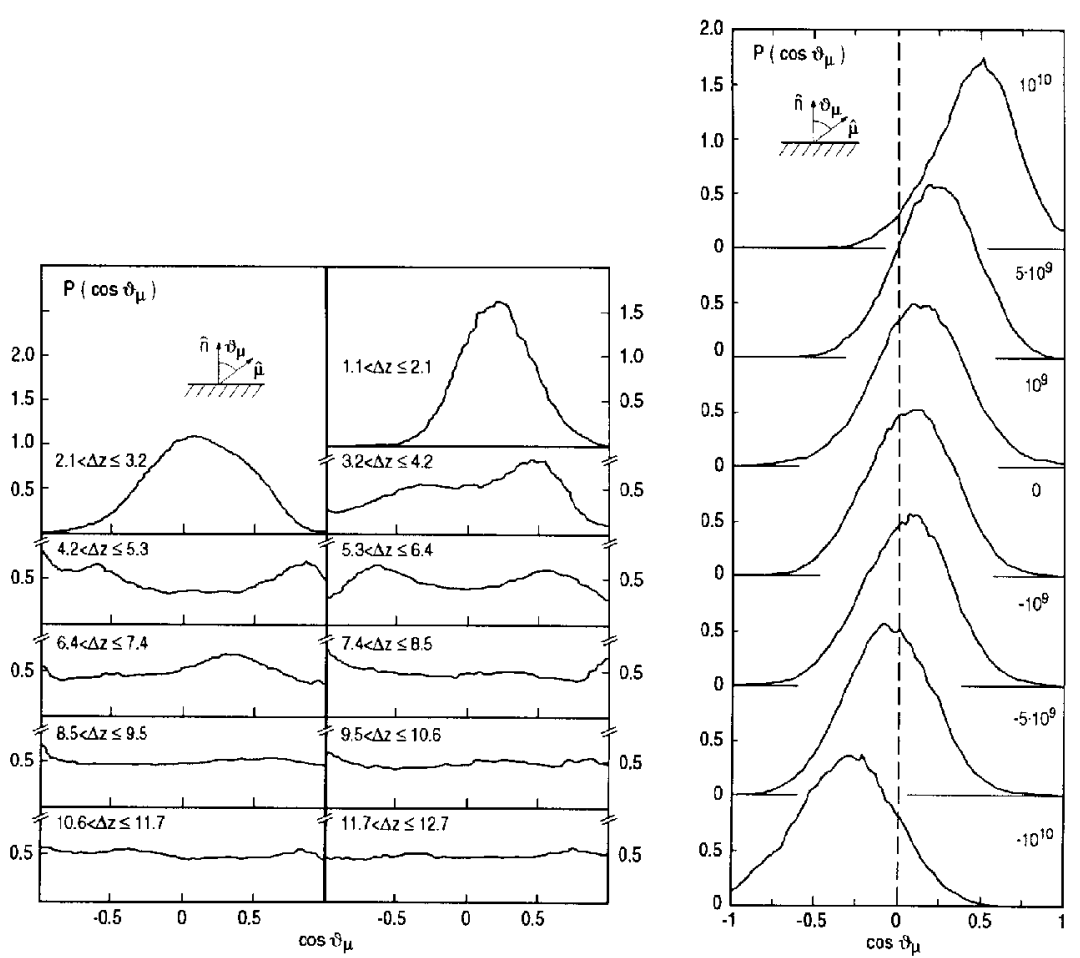

Figure 7. Distributions of $\cos \vartheta_{m} u$ for different distance ranges $\Delta z$ from the $\mathrm{Pt}(100)$ surface (left) and for a water monolayer for different strengths of an applied external electrical field (right). The field strengths are given in $\mathrm{V} / \mathrm{m}$ and $\Delta z$ in $\AA$. $\vartheta_{\mu}$ is defined in the insertion.

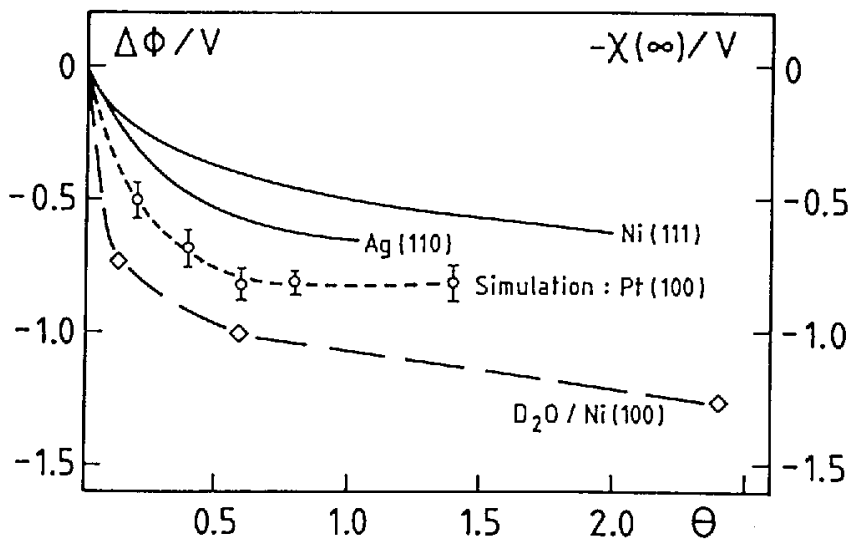

Figure 8. Comparison of the measured potential drop for various metals with that calculated from the simulation for the $\mathrm{Pt}(100)$ surface as a function of the degree of coverage [2].
It is obvious from this figure that a significant preferential orientation is found only for water molecules in the adsorbed layer [3]. The dipole moment vector points slightly away from the surface. Just as in the case of the density profile the orientation of water molecules is no longer influenced by the surface for distances larger than 9 A. 
The orientation of water molecules depends on the strength of an applied external electrical field. The results of simulations of a water monolayer on the $\operatorname{Pt}(100)$ surface are shown for various strengths of the electrical field also in figure 7 . The potential drops calculated from these distributions of $\cos \vartheta_{\mu}$ according to equation (1) show a linear dependence of $\chi$ on the field strength. The potential drop becomes zero for a field strength of about $2.5 \cdot 10^{9} \mathrm{~V} / \mathrm{m}[5]$.

The potential drop resulting from the orientations according to equation (1) agrees in the limits of error with the experimental value for the $\operatorname{Pt}(100)$ surface. But the agreement goes further as can be seen from figure 8 . There it is demonstrated how the potential drop depends on the degree of coverage of the metal surface. This dependence has not been measured for the $\operatorname{Pt}(100)$ surfaces but the simulation shows a similar dependence as determined experimentally for other metals. It seems, therefore, that the potentials derived from the quantum mechanical calculations describe the structure of water near the surface correctly [2].

Based on these results it can be expected that the influence of the $\mathrm{Pt}(100)$ surface on the ions and their hydration shells can be calculated from the simulation with a high degree of reliability. For the results reported here a water molecule was replaced either by a $\mathrm{Li}^{+}$or an $\mathrm{I}^{-}$in the adsorbed layer, instead of a simulation of a LiI solution between two platinum walls. During the simulation time of about $20 \mathrm{ps}$ the ions did not leave the adsorbed layer.

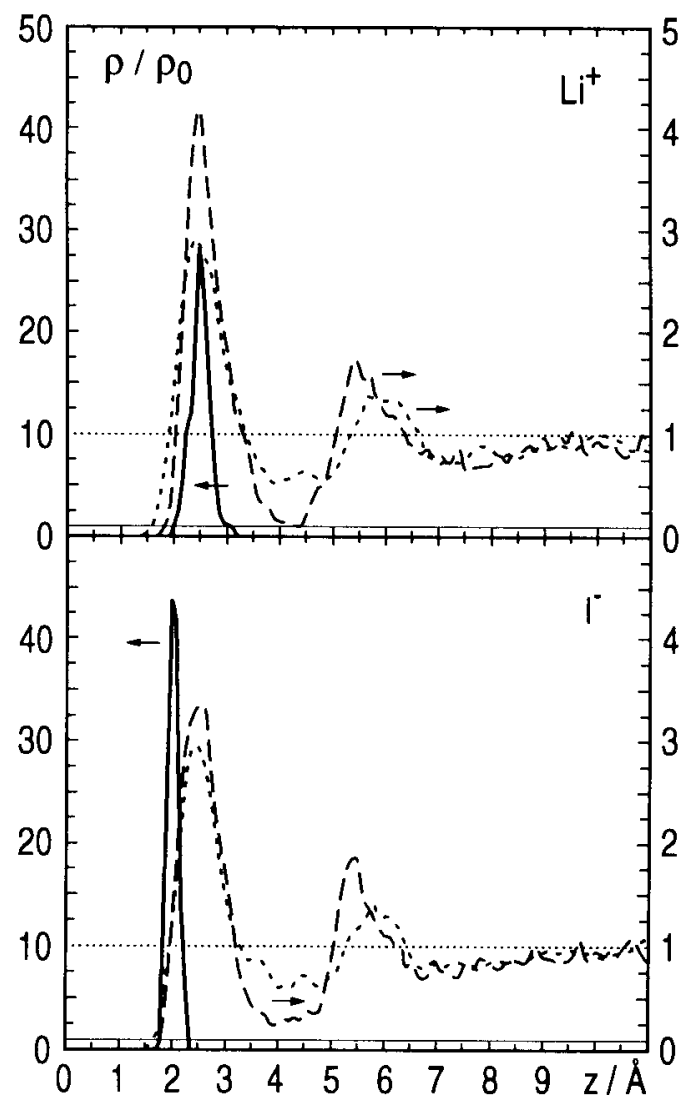

Figure 9. Normalized ion ((- -) and hydrogen (- -) atom densities as functions of distance from the $\operatorname{Pt}(100)$ surface, calculated from simulations with a lithium or an iodide ion in the boundary layer. The arrows indicate the relevant scale [10].

\section{This finding can be read from}

figure 9 where the density profiles for both ions are shown. For comparison also the profiles for the $\mathrm{O}$ and $\mathrm{H}$ atoms are depicted as dashed lines. They are not significantly different from that for pure water at the surface (figure $6)$.

The $\mathrm{I}^{-}$profile extends only over a very narrow distance range $1.8<$ $z<2.4 \AA$. There is no water molecule between the $\mathrm{I}^{-}$and the surface. Its maximum at $2.0 \AA$ coincides with the potential minimum for a position of the iodide ion opposite to a hollow site of the $\operatorname{Pt}(100)$ surface (figure 4), and it is obviously not influenced by the $\mathrm{I}^{-}$-water interactions. Therefore, the $\mathrm{I}^{-}$can be called "contact adsorbed" on the $\mathrm{Pt}(100)$ surface. 
The density profile of $\mathrm{Li}^{+}$is quite different from that of $\mathrm{I}^{-}$. In spite of the smaller size of the lithium ion the range of distances from the surface, $2.0<z<3.2 \AA$, is almost completely beyond that for the iodide ion and is much broader. The maxima of the $\mathrm{O}$ - and $\mathrm{H}$-atom profiles coincide with that of $\mathrm{Li}^{+}$at $2.5 \AA$. It can be seen from figure 4 that at this distance the potential energy of $\mathrm{Li}^{+}$is independent of its position relative to the platinum atoms of the surface layer. Very different from $\mathrm{I}^{-}$, it is obvious that both the distance range of $\mathrm{Li}^{+}$from the surface and its position relative to the surface platinum atoms is determined by the $\mathrm{Li}^{+}$-water and not by the $\mathrm{Li}^{+}-$ $\mathrm{Pt}(100)$ surface interactions. Therefore, it is justified to say that the $\mathrm{Li}^{+}$is "not contact adsorbed", although there is again no water molecule between the ion and the surface.

\subsection{Pair correlation functions}

The $\mathrm{O}-\mathrm{O}, \mathrm{Li}^{+}-\mathrm{O}$ and $\mathrm{I}^{-} \mathrm{O}$ pair correlation functions, depicted in figure 10 (left), show that the enhancement of the hydration shell structure of $\mathrm{Li}^{+}$
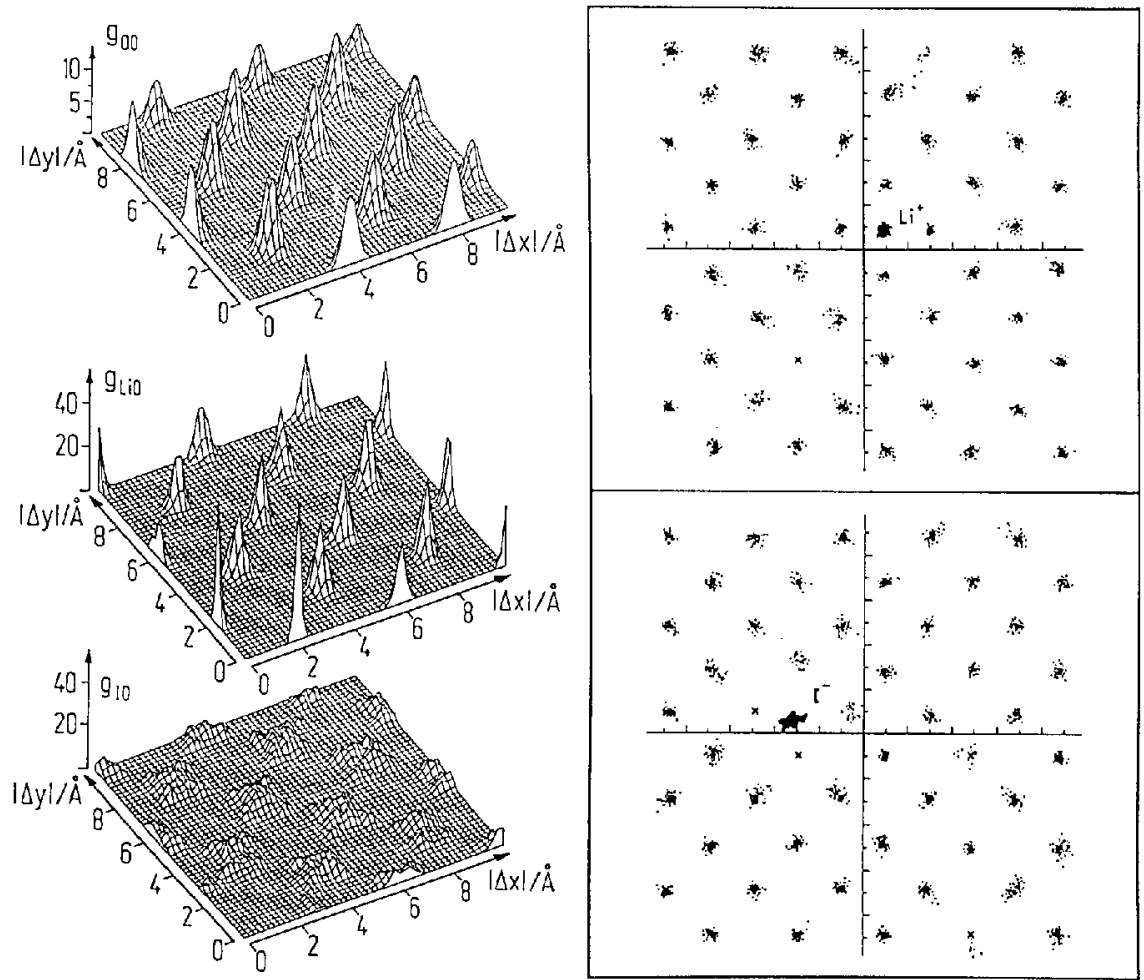

Figure 10. Oxygen-oxygen, lithium-oxygen and iodide-oxygen pair correlation functions in the adsorbed layer $(\Delta z \leqslant 4.2 \AA$ for all particles). $\Delta x$ and $\Delta y$ are the projections of the interatomic distance on the $\mathrm{x}$ and $\mathrm{y}$ directions of the laboratory coordinate system (left). Projection of the trajectories of the lithium, the iodide ion, and the oxygen atoms onto the $x y$-plane. The positions are marked by a dot every 0.05 ps. Only oxygen atoms of the adsorbed water layer are taken into account, and platinum atoms which are not covered with a water molecule are denoted by a cross (right) [10]. 
and the decrease for $\mathrm{I}^{-}$in the surface layer results from the formation of a pronounced quadratic water overlayer with a lattice constant of the $\operatorname{Pt}(100)$ surface of $2.77 \AA$. The origin $(\Delta x=0, \Delta y=0)$ coincides with the changing position of an $\mathrm{O}, \mathrm{Li}^{+}$or an $\mathrm{I}^{-}$in the surface layer $(z<4.2 \AA)$, and $|\Delta x|$ and $|\Delta y|$ are absolute values of the projections of distances of the $\mathrm{O}$-atoms in the surface layer from these reference particles. Both the reference particle and the $\mathrm{O}$-atoms referred to, move with respect to the Pi-lattice, and the shape of the peaks results from both movements.

The plot of $g_{O O}$ reflects the positions of the oxygen atoms on top of the platinum atoms, and the pronounced form of the peaks refers to their relatively small displacement. The sharper peaks for $g_{L i O}$ show that the displacements of $\mathrm{Li}^{+}$at its hollow site are smaller than those of $\mathrm{O}$ at its top, and the significantly broader ones for $g_{I O}$ compared with $g_{O O}$ show that the displacements of $\mathrm{I}^{-}$are much larger than those of $\mathrm{O}$. Indeed, the $\mathrm{I}^{-}$moves in a relatively widespread area between a hollow and a bridge site.

This can be seen from the right side of figure 10 where the trajectories of $\mathrm{Li}^{+}$and $\mathrm{I}^{-}$are drawn and where the $x$ - and $y$-coordinates of the oxygen atoms of the water molecules with $z<4.2 \AA$ are marked by a dot after every $0.05 \mathrm{ps}$. The positions of those $\mathrm{Pt}$ atoms which are not covered by oxygen atoms are indicated by crosses. Over the whole simulation time of $20 \mathrm{ps}$ the $\mathrm{Li}^{+}$remains very near to the hollow site of the $\mathrm{Pt}(100)$ surface at $x, y=1$. In accordance with the pair correlation functions the distributions of the neighbouring oxygen atoms above their respective Pt atoms are very narrow. The larger $\mathrm{I}^{-}$moves during this time in the area $-4.0<x<-2.5$ $\AA$ and $0<y<1.0 \AA$. It cannot occupy a hollow site as the first neighbour $\mathrm{I}^{-}$-O-distance is about $3.4 \AA$. In accordance with the energy curves in figure 4 it prefers positions between a hollow and a bridge site. Necessarily, two of the four neighbouring $\mathrm{Pt}$ atoms are not covered by oxygen atoms while their distributions around other $\mathrm{Pt}$ atoms are broader than in the $\mathrm{Li}^{+}$case.

\subsection{Hydration shell structure}

Geometrical arrangement of the oxygen atoms in the first hydration shells of ions can be deduced from the simulation by calculating the distribution of $\cos \vartheta$, where $\vartheta$ is defined as the O-Ion-O angle. The result is depicted in figure 11 for both ions in the boundary layer and in a bulk solution. The corresponding running integration numbers for $\cos \vartheta$, are shown additionally.

The distribution of $\cos \vartheta$ for $\mathrm{Li}^{+}$demonstrates clearly a strong preference for an octahedral symmetry in both cases. $P(\cos \vartheta)$ is narrower around 0 and -1 for the boundary layer in accordance with the well-defined nearest neighbour oxygen atom positions around $\mathrm{Li}^{+}$in the quadratic water overlayer (figure 10). The hydration number of $\mathrm{Li}^{+}$is reduced by about one in the boundary layer. As the octahedral plane of the hydration shell of $\mathrm{Li}^{+}$ parallel to the surface is fully occupied, the excluded volume of the surface is responsible for the missing water molecule (see also figure 9). The fifth water molecule belongs to the second water layer and is placed on top of the ion.

It can also be seen from figure 11 that neither in the bulk nor in the boundary layer a symmetry is recognizable, for the hydration shell of $\mathrm{I}^{-}$. $P(\cos \vartheta)$ is practically uniform over the whole range except for the excluded volume effect for $\cos \vartheta>0.8$ which results from the finite size of the water molecules. As the $\mathrm{I}^{-}$is contact adsorbed, the excluded volume effect of the $\mathrm{Pt}$ surface amounts to almost one half of the hydration shell and is responsible for the reduction of the hydration number of $\mathrm{I}^{-}$from 9.2 in the 
bulk solution to 5.1 in the boundary layer.

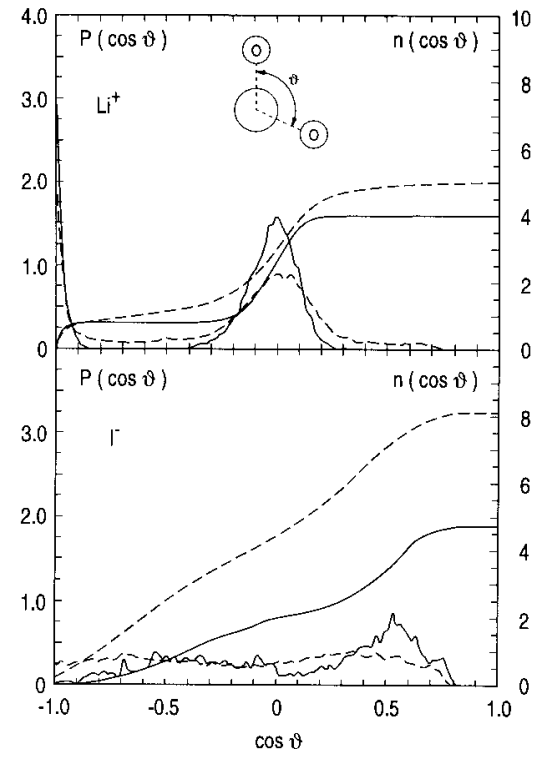

Figure 11. Normalized distributions of $\cos \vartheta$ - where $\vartheta$ is defined in the insertion for the water molecules in the first hydration shells of $\mathrm{Li}^{+}$and $\mathrm{I}^{-}$in the boundary layer (full) and in a 2.2 molal bulk solution (dashed). The corresponding running integration numbers are shown additionally [10].

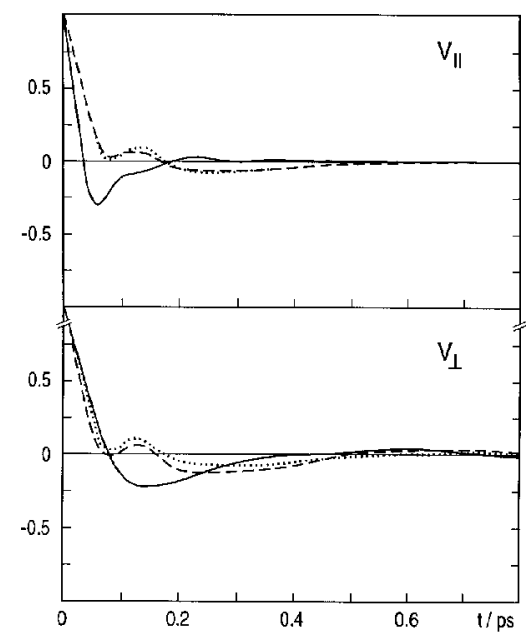

Figure 12. Center-of-mass velocity autocorrelation functions of water for the motions parallel (top) and perpendicular (bottom) to the surface for three different layers, defined by $\Delta z \leqslant 4.2 \AA$ (full), $4.2<z \leqslant$ $6.2 \AA$ (dashed), and $6.2<\Delta z$ (dotted) where $\Delta z$ denotes the distance of oxygen atoms from the surface [4].

\subsection{Self-diffusion coefficients}

The self-diffusion coefficients have been calculated from the velocity autocorrelation functions with the help of the Green-Kubo relation:

$$
D=\lim _{t \rightarrow \infty} \frac{1}{3} \int_{0}^{t} \mathrm{~d} t^{\prime}\left\langle\vec{v}(0) \cdot \vec{v}\left(t^{\prime}\right)\right\rangle .
$$

The averages have been calculated from the simulation by:

$$
\langle\vec{v}(0) \cdot \vec{v}(t)\rangle=\frac{1}{N_{T} N} \sum_{i=1}^{N_{T}} \sum_{j=1}^{N} \vec{v}_{j}\left(t_{i}\right) \cdot \vec{v}_{j}\left(t_{i}+t\right),
$$

where $N$ denotes the number of particles, $N_{T}$ the number of time averages and $\vec{v}_{j}(t)$ the velocity of particle $j$ at time $t$.

It seems appropriate for the investigations of the dynamical properties of electrolyte solution near the platinum surface to calculate the velocity 
autocorrelation functions separately for the components parallel and perpendicular to the surface. They are depicted in figure 12 for the three water subsystems adsorbed layer $(z \leqslant 4.2 \AA)$, second layer $(4.2<z \leqslant 6.2 \AA)$ and bulk region $(6.2<z)$. The figure shows that the difference between the second layer and bulk region is relatively small. The following discussion is, therefore, restricted to the difference between adsorbed layer and bulk region. The same is true for ions. Their velocity autocorrelation functions are depicted in figure 14 .

The self-diffusion coefficients for water calculated from. the velocity autocorrelation functions (figure 12) according to equation (2) are in the adsorbed layer in both directions by about a factor of ten smaller than in the bulk. With such small values for self-diffusion coefficients much longer simulations would be necessary to get more accurate results.

\subsection{Hindered translational motions}

The Fourier transformations of the velocity autocorrelation functions for water molecules (figure 12) lead to spectral densities of the hindered translational motions. They are depicted in figure 13. In order to understand the difference between the adsorbed layer and the bulk region and between the motions parallel and perpendicular to the surface it is necessary to keep in mind the assignment of frequencies. In bulk water the frequencies in the range up to about $100 \mathrm{~cm}^{-1}$ are attributed to the $\mathrm{O}-\mathrm{O}-\mathrm{O}$ bending and the higher ones to the $\mathrm{O}-\mathrm{O}$ stretching motions of water molecules [12].

The motions of water molecules parallel to the surface are much stronger hindered in the adsorbed layer than in the bulk. This is quite obvious from the $\mathrm{O}-\mathrm{O}$ pair correlation functions and the trajectories as depicted in figure 10 . Consequently, the frequencies of the $\mathrm{O}$ O stretching motions show a significant blue shift, as can be seen from figure 13 . The contribution of the O-O-O bending motions to the spectral density is small. For the motions perpendicular to the surface an almost opposite behaviour is found. Here the bending motions are dominant in the adsorbed layer and are shifted to higher frequencies because of the strong interaction of the water molecules with the $\operatorname{Pt}(100)$ surface. Accordingly, the intensity of the stretching motions is strongly reduced. The Pt(100) surface has only a small influence on the vibrations and intramolecular vibrations of water molecules in the adsorbed layer. Therefore, they will not be discussed here [4].

The velocity autocorrelation functions for the two ions have again been calculated for the particles located in the

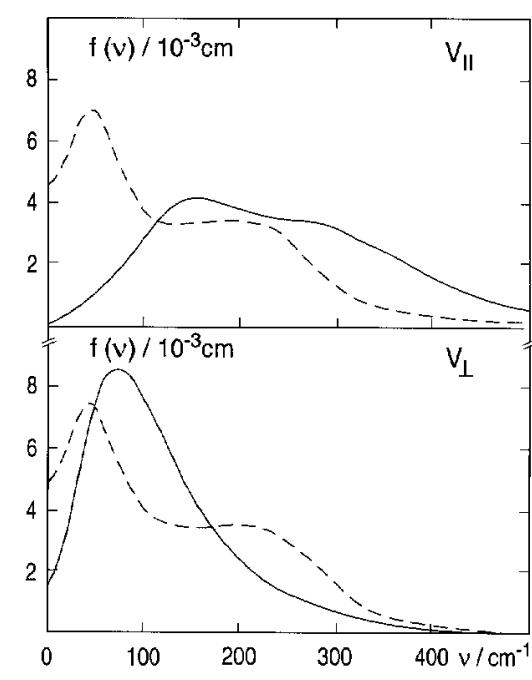

Figure 13. Spectral densities of the hindered translational motions of water molecules in the adsorbed layer (full) and in the bulk region (dashed) calculated separately for the motions parallel (top) and perpendicular (bottom) to the Pt(100) surface. boundary layer and in the bulk region and separately for the motions parallel and perpendicular to the surface. They are depicted in figure 14. The corresponding spectral densities are shown in the insertions [8]. 

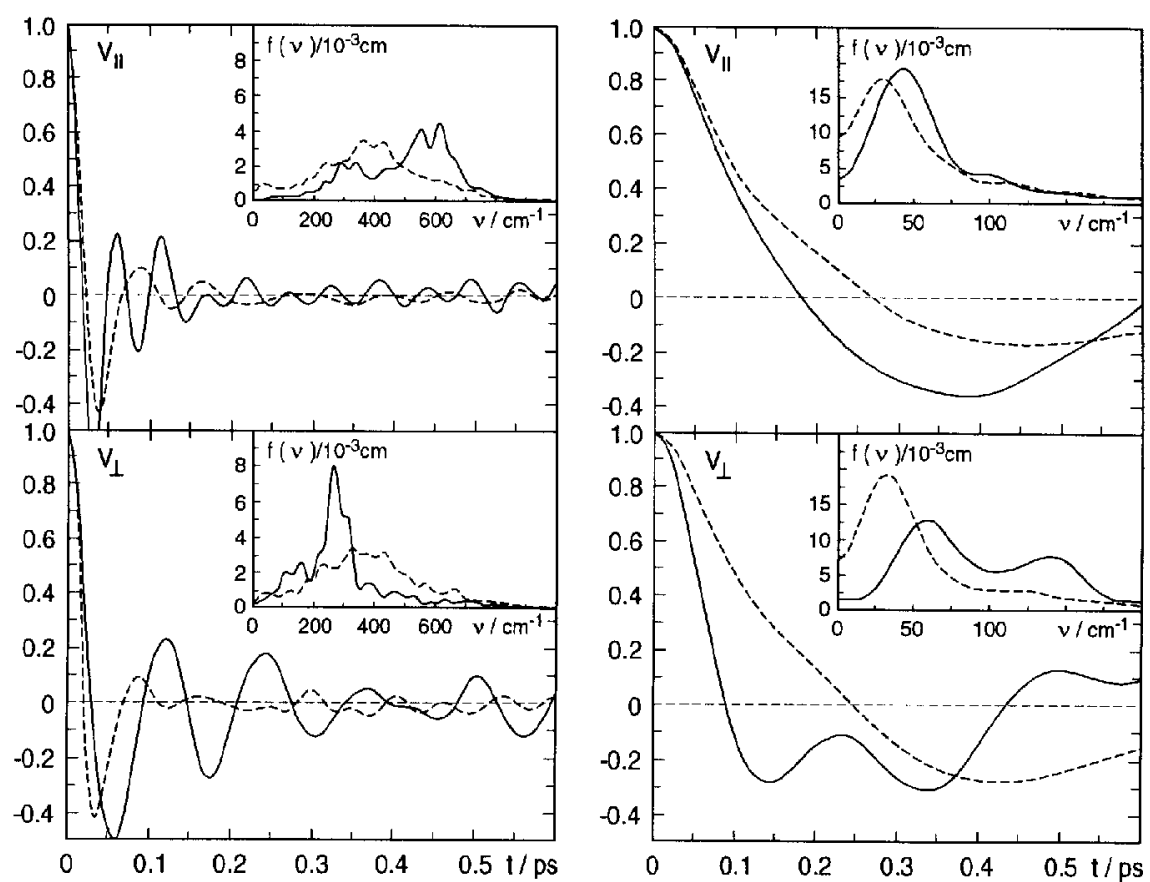

Figure 14. Normalized velocity autocorrelation functions and spectral densities of the lithium (left) and iodide ions (right) in the boundary layer (- $)$ and in the bulk region (- - ), calculated separately for the motions parallel (top) and perpendicular (bottom) to the Pt(100) surface.

The motions of the two ions in the boundary layer parallel and perpendicular to the platinum surface are quite different from those in the isotropic solution. The frequencies of the hindered translational motions of the lithium ion parallel to the surface show a strong blueshift. This reflects the enhanced $\mathrm{Li}^{+}$-water interactions as a result of the reduced water mobility, which is a consequence of the formation of the quadratic water overlayer. The motions of $\mathrm{Li}^{+}$perpendicular to the surface are characterized by a shift of the main peak in the spectral density to lower frequencies. This is a consequence of the larger mobility of the $\mathrm{Li}^{+}$because of the missing water molecule between the ion and the surface.

Different results are found for the iodide ion adsorbed on the platinum surface. The blue shift of the frequency maximum for the transverse motions of the ion in the boundary region is only about $20 \mathrm{~cm}^{-1}$. This indicates a movement of the heavy ion parallel to the surface which is only slightly disturbed by collisions with neighbouring water molecules adsorbed at the $\mathrm{Pt}(100)$ surface (figure 10). The perpendicular motions show a blue shift of the frequencies of the two main peaks, which follows from the strong interaction between the surface and the $\mathrm{I}^{-}$positioned near the energy minimum.

\section{Acknowledgements}

Financial support by Deutsche Forschungsgemeinschaft is gratefully acknowledged. 


\title{
References
}

[1] Holloway S., Bennemann K.H. Study of water adsorption on metal surfaces. // Surf. Sci., 1980, vol. 101, p. 327-333.

[2] Spohr S., Heinzinger K. A Molecular Dynamics study on the water/metal surface. // Ber. Bunsenges. Phys. Chem., 1988, vol. 92, p. 1358-1363.

[3] Spohr E. Computer simulation of the water/platinum interface. // J. Phys. Chem., 1989, vol. 93, p. 6171-6180.

[4] Spohr E. Computer simulation of the water/platinum interface. Dynamical results. // Chem. Phys., 1990, vol. 141, p. 87-94.

[5] Nagy G., Heinzinger K. A Molecular Dynamics study of water monolayers on charged platinum walls. // J. Electroanal. Chem., 1992, vol. 327, p. 25-30.

[6] Heinzinger K. Molecular Dynamics of water at interfaces. In: Structure of electrified interfaces (eds. Lipkowski J., Ross P.N.). Frontiers of electrochemistry, vol. II, VCH Publishers, New York, 1993.

[7] Seitz-Beywl J., Poxleitner M., Probst M.M., Heinzinger K. On the interaction of ions with a platinum metal interface. // Int. J. Quantum Chem., 1992, vol. 42, p. 1141-1147.

[8] Heinzinger K., Seitz-Beywl J., Poxleitner M. Molecular Dynamics simulations of platinum-aqueous electrolyte solution interfaces. In: Microscopic models of electrode-electrolyte interfaces (eds. Halley J.W., Blum L,), Electrochem. Soc. Proceedings Series, 1993, vol. 93-5.

[9] Bopp P., Jancso G., Heinzinger K. An improved potential for non-rigid water molecules in the liquid phase. // Chem. Phys. Lett., 1983, vol. 98, p. 129-134.

[10] Seitz-Beywl J., Poxleitner M., Heinzinger K. A Molecular Dynamics study of ionic hydration near a platinum surface. // Z. Naturforsch., 1991, vol. 46a, p. $876-886$.

[11] Allen M.P., Tildesley D.J. Computer simulation of liquids. Clarendon Press, Oxford, 1989.

[12] Sceats M.G., Rice S.A. The water-water pair potential near the hydrogen bonded equilibrium configuration. // J. Chem. Phys., 1980, vol. 72, p. 32363247 .

\section{ІОННА ГІДРАТАЦІЯ БІЛЯ ПЛАТИНОВӦ̈ ПOBEPXНI $(100)$}

\author{
К.Гайнщінгер
}

При моделюванні методом молекулярної динаміки, яке представлене тут, використовується гнучка модель БоппаЙанчо-Гайнцінгера для опису взаємодії вода-вода. Потенціали взаємодії між іонами і водою, між поверхнею платини і водою та між поверхнею платини і іонами базуються на розрахунках ab initio. Показано, що структурні і динамічні властивості іонів та їх гідратаційні оболонки у шарі, адсорбованому на поверхні $\operatorname{Pt}(100)$, значно відрізняються від об'ємних. Але вплив металу на ці властивості обмежений практично адсорбованим шаром. 